\title{
Coupe du Monde 2014 au Brésil : deux facettes de la prostitution (Belo Horizonte e Recife)
}

\author{
2014 Football World Cup: \\ two facets of prostitution (Belo Horizonte and Recife)
}

\begin{abstract}
César Teixeira Castilho
Universidade Federal de São João del-Rei (UFSJ), São João del-Rei / Brasil Doutor em Sciences du Sport, de la Motricité et du M. Humain, Université Paris-Sud 11 (UFR-STAPS) castcesarster@gmail.com
\end{abstract}

Dominique Charrier Maître de Conférences Université Paris-Sud 11 (UFR-STAPS), Orsay / França Doutor em Sciences Économiques (Université Paris 9)

\author{
Barbara Evrard \\ Maître de Conférences Université de Rouen-Normandie (UR) / França \\ Doutora em Sciences du Sport (STAPS-Université de Rouen)
}

RÉSUMÉ : Cette étude présente une analyse sur la prostitution dans le cadre de la Coupe du monde de football 2014 au Brésil, au travers des projets distincts mis en place dans deux villes d'accueil : Belo Horizonte (Sud-est) et Recife (Nord-est). Par le biais d'une approche qualitative - entretiens, observations et photographies - ce thème est exposé en mettant l'accent sur les aspects sociaux et la participation des groupes exclus à l'égard des événements sportifs. Bien que, à court terme, les résultats des projets soient satisfaisants, à long terme, des mesures socio-éducatives effectives doivent être priorisées pour améliorer la situation des acteurs impliqués. Tant la question de la prostitution, que le tourisme sexuel infantile, doivent être débattus davantage à la fois dans la société et dans le monde académique.

MoTS-CLÉS : Coupe du monde 2014; Les aspects sociaux; La prostitution.

ABSTRACT: This study aims to analyze the issue of prostitution in Brazil from the implementation of two different social projects carried out in the host cities of Belo Horizonte (Southeast region) and Recife (Northeast) in the context of the World Cup Football FIFA 2014. Through an approach qualitative - interviews, observations and photographs - the question of prostitution will be analyzed taking into account the social aspects entangled, and the implications of excluded sectors of society in the organization of so-called mega-events. Although in the short term, the results obtained in the analyzed projects have been satisfactory, in the long run, effective social and educational measures must be prioritized to improve the situation of the actors involved. Both the issues of prostitution, as well as the child sexual tourism, studied complain for a more active debate in society and academic world.

KEYWORDS: 2014 World Cup; The social aspects; Prostitution. 
FuLiA / UFMG - [...] deux facettes de la prostitution

\section{INTRODUCTION}

Le 30 octobre 2007, la Fédération internationale de football association (FIFA) a officialisé le choix du Brésil comme pays hôte de la Coupe du monde (CM) de football 2014. L'une grande fête a été organisée par le gouvernement afin de célébrer cette conquête. La population était d'autant plus heureuse que le football joue en rôle majeur dans le contexte social du pays, il est considéré comme la principale activité sportive des Brésiliens. ${ }^{1}$ La culture footballistique est dans l'imaginaire des brésiliens et la réussite finale de cet état d'esprit ne pouvait être que l'accueil d'un nouveau Mondial. Certes, le pays entier était en faveur de l'accueil de l'événement. Mais, pour quelques-uns plus critiques, le défi de l'organisation paraissait trop difficile vis-à-vis des ennuis qui ont perturbé la nation brésilienne depuis les dernières années, tels que la corruption, la violence, les inégalités sociales, entre autres. En plus, au même moment, plusieurs médias internationaux ont publié des reportages mettant l'accent sur l'incapacité du pays à organiser un tel événement. ${ }^{2}$

En plus de l'accueil de la CM de football, le Brésil a été désigné le 2 octobre 2009 par Jacques Rogge, président du Comité international olympique (CIO), lors de la 121ํㅗㅇ session du CIO, comme le pays hôte des Jeux olympiques (JO) d'été 2016. Ce choix est venu durant le second mandat du président Lula ce qui a été associé à son caractère charismatique et à sa reconnaissance mondiale. À cette époque, le pays traversait une période de transition caractérisée par des changements socioéconomiques de grande ampleur. De cette façon, l'arrivée de ces deux grands événements était considérée comme la cerise sur le gâteau d'après le gouvernement lui-même. Cependant, au fil du temps, la situation envisagée s'est montrée en peu différente, surtout quand les travaux ont démarré et que certains signes de désordre ont commencé à être visibles.

\footnotetext{
${ }_{1}^{1}$ ALMEIDA; GUTIEREZ. O lazer no Brasil: do nacional-desenvolvimentismo à globalização.

2 DAMO; OLIVEN. O Brasil no horizonte dos megaeventos esportivos de 2014 e 2016: sua cara, seus sócios e seus negócios.
} 
La CM de football, organisée par la FIFA, ainsi que les JO d'été, organisés par le CIO, font partie de ce qu'on appelle aujourd'hui de grands événements sportifs (GES). D’après Roberts ${ }^{3}$ ce qui définit un événement sportif comme " grand » ou « méga » sont sa discontinuité, son originalité, son caractère international et sa composition globale hors-norme. Ce que Roberts ${ }^{4}$ désigne comme " grand » est en relation directe avec la capacité de ces événements à atteindre des millions de personnes à travers le monde via la télévision ou d'autres moyens de communication. Ce concept de « grand » a attiré une audience de plus en plus internationale. Notamment, la CM 2002, organisée par le Japon et la Corée du Sud simultanément, a été transmise pendant plus de 41000 heures dans 213 pays ce qui a produit une audience cumulative de 28,8 milliards de téléspectateurs. ${ }^{5}$

Désormais, les enjeux économiques, à ce moment plus importants que la compétition sportive elle-même, ont commencé à prendre une dimension de plus en plus importante vis-à-vis des organisateurs de ces événements globaux. Le droit à l'image, payé surtout par les chaînes de télévision, a commencé à être exploité de plus en plus conduisant à des profits considérables à chaque édition de l'événement.

En plus de l'activité économique autour des GES, la communauté scientifique a commencé à se pencher sur d'autres aspects aussi importants comme ceux liés aux enjeux économiques. Par conséquent, on peut également souligner la parution d'études scientifiques pertinentes liées à la politique, à l'environnement, au contexte social, aux héritages à court et à long terme, et au tourisme. Ces nouvelles questions analytiques sont essentielles pour que notre compréhension de l'événement sportif soit toujours plus aigüe et donc plus critique. Dans la mesure où ces nouvelles analyses ont commencé à être mises en exergue, la multidisciplinarité a gagné du terrain empruntant de nouvelles connaissances aux sciences sociales, à

\footnotetext{
${ }^{3}$ ROBERTS. The leisure industries, p. 108.

${ }_{5}^{4}$ ROBERTS. The leisure industries.

${ }^{5}$ MADRIGAL; BEE; LABARGE. Using the Olympics and FIFA World Cup to enhance global brad equity, p. 182.
} 
FuLiA / UFMG - [...] deux facettes de la prostitution

l'anthropologie, à l'économie, à l'éducation physique, au tourisme, à la biologie, l'architecture, entre autres.

\section{LES IMPACTS SOCIAUX ET LES HYPOTHESES DE L'ETUDE}

Les grands événements sportifs sont considérés comme étant des composantes importantes pour l'économie locale et leur organisation peut être bénéfique au regard de plusieurs aspects pour l'ensemble de la ville d'accueil, ou du pays hôte. À en croire le nombre de villes qui se portent candidates à l'organisation des J0, aussi bien d'hiver que d'été, l'accueil d'un GES constituerait aujourd'hui une bonne affaire pour la ville organisatrice, la région, voire pour le pays entier. Neuf villes ont ainsi officiellement fait acte de candidature à l'organisation des JO d'été de 2012, et sept villes étaient initialement en course pour accueillir les JO d'hiver de $2014 .^{6}$

En plus d'une couverture médiatique internationale du pays ou de la ville d'accueil, de nombreux travaux attestent de la diversité des effets locaux des GES.7 Qu'ils soient politiques (notoriété accrue, construction d'une image positive pour la collectivité locale ou pour les élus, etc.), économiques (production et consommation de richesses, créations nettes d'emplois, aménagement du territoire, etc.), sociaux (cohésion sociale, valorisation du territoire et de ses habitants, animation, prévention ou insertion de publics spécifiques, etc.), sportifs (découverte et développement d'une pratique sportive, consolidation des structures de l'offre, etc.) ou encore médiatiques (retransmissions locales sur grands écrans, citations dans des articles et reportages, etc.), sont également des composants de l'attractivité de ces manifestions sportives majeurs. ${ }^{8}$

Dans cette étude, dans le cadre de la CM 2014 au Brésil, on se penche sur ces impacts, plus spécifiquement, les impacts sociaux liés aux populations vulnérables du

\footnotetext{
${ }_{7}^{6}$ JUNOD. Grands événements sportifs: des impacts multiples.

7 BARGET; GOUGUET. L'accueil des grands événements sportifs: quel impact économique ou quelle utilité sociale pour les régions ? : l'exemple de la Coupe du monde de Rugby 2007 en France. CHARRIER; JORDAN. L'impact touristique local des grands événements sportifs: une approche qualitative de la Coupe du monde de Rugby en île-de-France. WAITT. Social impacts of the Sidney Olympics

${ }^{8}$ CHARRIER; JORDAN. L'impact touristique local des grands événements sportifs: une approche qualitative de la Coupe du monde de Rugby en Île-de-France, p. 46.
} 
FuLiA / UFMG - [...] deux facettes de la prostitution

pays hôte. Il n'existe pas de consensus quant à la définition des impacts sociaux. Olsen et Merwin ${ }^{9}$ les comprennent comme " des changements dans la structure et le fonctionnement des modes de contrôle social qui se produisent en conjonction avec une innovation ou modification environnementale, technologique ou sociale ». Mathieson et Wall ${ }^{10}$ se réfèrent aux impacts sociaux comme « des changements dans la qualité de vie des résidents des destinations touristiques ». Alors que la première définition fournit à la fois une description des impacts sociaux et les raisons de leur présence en termes d'influences environnementales, technologiques et sociales, le dernier montre la relation entre les implications sociales et le tourisme, mais ne parvient pas à expliquer pourquoi ces impacts se produisent et comment ces changements dans la qualité de vie peuvent être acquis.

Cet écart est en partie repris par Hall ${ }^{11}$ qui voit les impacts sociaux comme « la manière dont le tourisme et les différents modes de voyage perturbent un système de valeurs collectives ou individuelles produisant des changements au niveau des comportements, des structures communautaires, des styles de vie et de la qualité de vie ». Or, en prenant cette définition, les changements prévus par l'arrivée d'un événement doivent être compris à partir d'une perspective ample car toutes les couches sociales et toutes les institutions seront affectées, tant à court qu'à long terme. En outre, une préparation préalable de la société en générale, au-delà des organisateurs officiels, a lieu puisque cette opportunité n'arrive qu'à de rares occasions. Au Brésil, la dernière CM s'est produite en 1950.

Étant donné que la situation social brésilienne, malgré des avancées sensibles depuis les années 2000, est encore l'une des plus fragiles au monde, ${ }^{12}$ l'analyse de ces impacts peut éclaircir l'importance accordées par les élus à l'organisation d'un tel événement. De plus, cela nous révèle comment les différents groupes - tant les

\footnotetext{
${ }^{9}$ OLSEN; MERWIN. Towards a methodology of conducting social impacts assesments using quality of life indicators.

${ }^{11}$ MATHIESON; WALL. Tourism: Economic, Physical and Social Impacts.

${ }_{11}^{11}$ HALL. Adventure, sport and health tourism, p. 67.

12 Le Brésil est actuellement parmi les dix pays les plus inégaux socialement selon une enquête établie par l'ONU.
} 
FuLiA / UFMG - [...] deux facettes de la prostitution

institutions autonomes, que les institutions publiques - s'organisent et quels sujets sont prioritaires à l'égard des aspects sociaux. Quoique la festivité soit vécue conjointement par les locaux et les touristes, après l'événement, ce sont les locaux qui seront les plus affectés par les héritages.

Parmi les impacts sociaux discutés dans la littérature académique, on trouve souvent une tendance à les classer comme positifs ou négatifs. ${ }^{13}$ Certes, ce tri apporte une compréhension plus pédagogique concernant l'exposition de résultats scientifiques, néanmoins, cette attitude révèle à la fois un jugement de valeur entre ce qui était établi comme bien et mal. À ce sujet, en analysant certaines études, ${ }^{14}$ on trouve la thématique de la prostitution, quand elle apparaît, dans le tableau concernant les aspects négatifs. Bien que pour certains groupes impliqués, comme les habitants locaux, cela puisse être vu comme une nuisance, des points positifs sont aussi importants à souligner. À cet égard, la question doit être posée à ceux qui sont affectés directement, notamment les prostituées elles-mêmes et d'autres acteurs, comme les hommes politiques, qui ont également intérêt à débattre ce thème vis-à-vis de la CM 2014. Rien de tel qu'une pareille opportunité médiatique pour discuter d'un sujet à la fois controversé et intrigant socialement.

De cette façon, cette étude cherche à effectuer une analyse aigüe sur deux projets dans le cadre de la CM 2014, dans deux villes d'accueil différentes - Belo Horizonte (BH) et Recife - prenant la prostitution comme axe principal. Ainsi, deux projets ont été choisis : à Belo Horizonte, le projet intitulé « cours de langues pour les prostituées » organisé par l'Association des prostituées de la ville et, à Recife, la politique publique mise en place par la municipalité afin de combattre le tourisme sexuel infantile dans la région. Depuis ces deux modèles, un élargissement du débat sur ce propos est possible dans la mesure où les acteurs principaux sont de provenances distinctes, l'un lié à une association privée et, l'autre, aux pouvoirs

\footnotetext{
${ }^{13}$ FREDLINE. Host and Guest Relations and Sport Tourism. HALL. Adventure, sport and health tourism. GETZ. Festivals, Special Events and Tourism. RITCHIE. Assessing the impact of hallmark events: conceptual and reserach issues. HIGHAM. Sport as an Avenue of Tourism Development: An Analysis of the Positive and Negative Impacts of Sport Tourism.

${ }^{14}$ GETZ. Festivals, Special Events and Tourism. HALL. Adventure, sport and health tourism.
} 
FuLiA / UFMG - [...] deux facettes de la prostitution

publics. Dans un même événement, on perçoit des organisations particulières mais qui visent, d'une certaine manière, le but d'en profiter selon chaque cas.

La prostitution au Brésil n'est pas considérée comme un crime par les lois locales. En revanche, le proxénétisme et le trafic organisé autour de cette pratique le sont. En outre, actuellement, la question sur la légalisation des « professionnelles du sexe » est en pleine discussion vu qu'une loi est en train d'être votée par la Chambre des députés. Malgré le conservatisme d'une partie importante des élus du pays, on en retrouve quelques-uns qui sont prêts à se battre pour cette cause. ${ }^{15}$

D'un autre côté, la question relative au tourisme sexuel infantile a toujours frappé le pays, en particulier la région Nord-est, où se situe la ville de Recife. D’après les études sur ce thème, ${ }^{16} 40 \%$ des plaintes déposées viennent de cette région. Ainsi, pour l'arrivée de la CM 2014, les pouvoirs publics ont entamé une politique d'action préventive pour combattre cette pratique à la fois à travers une approche pédagogique avec les habitants locaux et une lutte déclarée contre ceux qui en profite économiquement. De plus, il était prévu une recherche minutieuse pour identifier les touristes (visiteurs) qui cherchaient ses services.

Dans ce contexte, cette étude prévoit de répondre à quelques questions concernant la prostitution dans le cadre de la CM 2014 à travers deux cas divers situés dans des villes d'accueil différentes. En tenant compte des aspects sociaux, comment peut-on mettre l'accent sur la population exclues dans le cadre des GES ? Quelles sont des particularités entre ces deux projets eu égard aux caractéristiques socioéducatives de chaque région?

À travers d'une recherche sur le terrain d'une durée de deux mois, deux années consécutives, on propose une discussion scientifique sur ce thème pour rendre possible un autre regard vis-à-vis des impacts sociaux des GES. Au Brésil, d'une manière générale, les inégalités sociales sont d'autant plus évidentes que les populations exclues - tels que les prostituées ou les victimes directes - se trouvent encore plus en marge dans une situation d'accueil d'un événement sportif majeur.

\footnotetext{
${ }^{15}$ MADER. Lei da prostituição divide a Câmara, p. 6.

${ }^{16}$ LOURENÇO; FONTES. Articulação e mobilização de parceiros.
} 
Toutefois, même à l'écart de la société, ces individus se font entendre, même si quelques-uns ne le veulent pas.

Ensuite, afin de bien débattre ces questions, on expose la méthodologie utilisée dans cette recherche et les principaux résultats analysés suite à la récolte des données au cours du terrain.

\section{METHODOLOGIE}

La première action avant la recherche sur le terrain a été la réalisation d'un tri de projets liés à la prostitution et au tourisme sexuel infantile dans le cadre de l'organisation de la CM 2014. Après une première sélection d'environ 12 projets à travers le pays, nous avons priorisé ceux qui se situaient dans des régions distinctes au niveau socio-économique. De cette façon, en tenant compte à la fois de l'originalité et de l'endroit des projets, on est arrivé aux régions Sud-est, pour le projet intitulé « cours des langues des prostituées », et la région Nord-est, pour celui nommé " prévention du tourisme sexuel infantile ». Parmi les villes d'accueil du Mondial, Belo Horizonte (Sud-est) et Recife (Nord-est) ont été choisies pour cette étude.

Les données de terrain ont été recueillies en trois moments différents par rapport à la CM 2014 - avant, pendant et après - entre les années 2013 et 2015, auprès des responsables de chaque projet, des hommes politiques et des habitants confrontés dans leur quotidien à la présence de prostitution ou du tourisme sexuel infantile. Dans les deux cas, la présence féminine s'est faite majoritaire : à Belo Horizonte c'est chiffre est de $100 \%$ puisque il s'agit d'une association de prostituées; et à Recife, les filles représentent $80 \%$ des plaintes déposées. ${ }^{17}$ Tous les noms ou surnoms de personnes ont été modifiés afin de préserver l'anonymat.

Le recueil des matériaux à combiné l'analyse des documents concernant ce sujet - soit les documents de la presse, soit les documents officiels -, l'observation sur site, sous leurs dimension spatiale et interactionnelle, la participation aux cours de

\footnotetext{
${ }^{17}$ LOURENÇO; FONTES. Articulação e mobilização de parceiros.
} 
langues promus par l'Association de prostituées de Belo Horizonte, et des visites dans les quartiers les plus affectés par le tourisme sexuel à Recife. Des entretiens approfondis ont été réalisés avec les personnes impliquées (porteurs des projets, hommes politiques liés à l'organisation et habitants locaux), ainsi que des conversations informelles avec les personnes moins compromises, mais qui pouvaient apporter des points de vues utiles. Cette dernière catégorie recouvre un contenu hétéroclite, recueilli auprès d'acteurs divers, tels que : supporters, visiteurs, locaux, journalistes, organisateurs, volontaires de l'événement, commerçants, etc.

L'aspect temporel, au-delà de l'aspect territorial, apporte des données majeures car l'un des objectifs de l'étude est exactement de débattre des impacts des projets sociaux après la fin de l'événement. Ainsi, l'observation du terrain et les entretiens approfondis ont été faits dans les mêmes endroits et avec les mêmes acteurs, respectivement. Le point de vue diffère à l'égard du moment analysé - avant, pendant et après - ce qui favorise la compréhension de ces projets à long terme dans ces régions.

En outre, la photographie a été utilisée comme outil de recherche afin d'illustrer certaines situations clés dans la portée de l'étude. Par exemple, à Belo Horizonte, les maisons closes décorées avec des drapeaux du Brésil, et d'autres équipes, faisant référence au Mondial; et à Recife, des affiches de prévention du tourisme sexuel déployées dans tout le centre-ville ce qui donnaient la dimension du projet. Pour bien comprendre la démarche effectuée, à suivre, on exposera les détails de la stratégie d'approche des acteurs dans chaque ville étudiée.

\section{BELO HORIZONTE}

Belo Horizonte est la plus grande ville de l'État brésilien du Minas Gerais dont elle est la capitale. La ville compte 2375444 habitants dans ses limites et plus de 5397438 habitants vivent dans son agglomération, ce qui en fait la troisième agglomération la plus peuplée du pays, après São Paulo et Rio de Janeiro. ${ }^{18}$ Elle a été inaugurée en 1897

\footnotetext{
${ }^{18}$ IBGE. Estimativas da população residente no Brasil e unidades da federação.
} 
et se situe dans la région Sud-est du pays. Il s'agit de la région la plus riche des cinq régions brésilienne. Les quatre états qui la composent (le São Paulo, le Minas Gerais, le de Rio de Janeiro et l'Espírito Santo) représentent environ 60\% du PIB brésilien. Centre commercial et industriel du Brésil, le Sud-est concentre notamment les institutions supérieures les plus importantes, ainsi que les principaux sièges d'entreprises nationales et internationales. L'économie se fonde sur les productions industrielles et agricole régionales. L'industrie automobile et l'industrie lourde y sont les plus importantes. Pour ce qui est de l'agriculture, les principaux produits sont : le café, la canne à sucre, le soja et les oranges.

Le projet créé par l'Association des prostituées de Belo Horizonte a été conçu pour répondre à une demande des prostituées adhérentes elles-mêmes. Cette association compte à présent plus de 4000 femmes et est considérée comme l'une des associations les plus organisées et engagées du pays. On y trouve des femmes originaires de plusieurs régions du pays, majoritairement issues des couches sociales faibles, qui y viennent en raison de la réputation de l'institution. La présidente de l'association est élue par les adhérentes tous les trois ans et, parmi ces responsabilités, il y a notamment une préoccupation par rapport à l'instruction de ses membres. De cette façon, plus que le profit économique (puisque l'apprentissage d'une langue étrangère aide à approcher les touristes), le projet visait également l'amélioration des compétences des travailleuses.

Les collectes des données concernant ce projet ont été entamées une année avant le Mondial durant la Coupe des confédérations 2013. À ce moment, d'importants rassemblements populaires ont eu lieu à travers le pays afin de critiquer l'organisation de la $\mathrm{CM}$ et les dépenses excessives vis-à-vis des constructions des stades. ${ }^{19}$ D’abord, une première approche a été réalisée en 2013 ce qui nous a permis d'accompagner le démarrage des cours qui ont eu lieu dans un centre commercial à proximité du quartier où les maisons closes se trouvent. En effet, à BH, c'est au centreville, dans deux rues approximativement, que se situe la plupart des maisons de

\footnotetext{
${ }^{19}$ SOUSA. Comprendre les récentes manifestations de rue.
} 
FuLiA / UFMG - [...] deux facettes de la prostitution

prostitution. À ce moment, les observations des cours de langues ont été accomplies, aussi bien que des entretiens avec les élèves concernées et les porteuses du projet, singulièrement la présidente Mme Bethânia.

Le deuxième moment du terrain a eu lieu quinze jours avant le coup d'envoi du Mondial et prévoyait l'analyse du moment actuel du projet en tenant compte de cette année de préparation déjà écoulée depuis 2013. En outre, à travers les outils de recherche, les progrès de l'enseignement et l'opinion des personnes impliquées ont pu être vérifiés. À moins de deux semaines de l'événement, le cours de langues était prévu de s'arrêter dans quelques jours parce que, durant le Mondial, l'enseignement ne fonctionnerait pas.

Le recueil des données à Belo Horizonte s'est accompli en 2015, exactement un an après la fin de la CM 2014. À ce moment, de nouvelles observations participantes et des entretiens ont été réalisés dans le but de comprendre le bilan final du projet et les impacts perçus par les personnes concernées. Les médias nationaux et étrangers ont publié plusieurs reportages sur l'impact après un an de l'événement mais, sans surprise, peu de place a été accordée aux impacts sociaux sur les habitants exclus. Néanmoins, malgré le succès de l'ambiance pendant la durée du Mondial partout, le bilan général est que le pays a énormément dépensé au niveau des infrastructures notamment pour les stades et les aéroports - mais, en revanche, n'a pas vraiment laissé un legs social important pour sa population. ${ }^{20}$

\section{RECIFE}

Recife est la capitale de l'état du Pernambouc. Elle est la neuvième plus grande ville nationale et son agglomération compte 3,7 millions d'habitants, la cinquième du pays. $^{21}$ C'est une des villes brésiliennes les plus anciennes, fondée en 1537, et représente à la fois un centre économique et touristique de la région Nord-est. En

\footnotetext{
${ }^{20}$ BBC. Un an après la Coupe du monde 2014, seulement la moitié de l'héritage promis a effectivement été livré à la population. AMORA. Um ano após a Copa do Mundo, 35 obras não estão prontas.

${ }^{21}$ IBGE. Estimativas da população residente no Brasil e unidades da federação.
} 
FuLiA / UFMG - [...] deux facettes de la prostitution

comparaison aves l'autres régions du Brésil, c'est la deuxième en terme de population, après celle du Sud-est, le troisième territoire en superficie, l'IDH le plus faible et le troisième PIB. ${ }^{22}$ Cette région est le berceau de la colonisation portugaise et sa population est formée à partir des trois racines : les indigènes, les européens et les afro-descends. Depuis l'époque de l'empereur D. Pedro II, XIXème siècle, la région souffre d'émigration massive vers le Sud-est. Cela est due aux hauts niveaux d'inégalités sociales renforcées par un problème de sècheresse. ${ }^{23}$

À cause des inégalités sociales considérables, aussi bien que d'un manque d'instruction de la plupart de la population, la région Nord-est - y compris la ville de Recife - est frappée par le tourisme sexuel infantile. ${ }^{24}$ Cette activité criminelle est la cible des politiques publiques depuis les années 1990 mais, malgré les efforts, est toujours plus ou moins présente. Même si ce type de prostitution est le seul choix pour une partie de la population de sortir d'une situation de pauvreté extrême, on trouve dans cette affaire un nombre considérable d'individus concernés qui harcèlent ces enfants pour profiter de leur fragilité. En outre, le tourisme sexuel possède un certain attrait dans le monde. De cette façon, plus que cibler les habitants locaux pour les renseigner sur ce genre d'abus sexuel, ces politique publiques cherchent également l'emprisonnement des personnes impliquées. ${ }^{25}$

Le recueil des données a été entamé à partir de lectures d'articles de la presse et des institutions publiques concernant cette thématique. Depuis la nomination du Brésil comme pays hôte en 2007, les organes publics - Ministère du tourisme, la Police fédérale et les municipalités de la région Nord-est - ont établi comme priorité la lutte contre le tourisme sexuel infantile dans le cadre de la CM 2014. Étant donné que cette festivité attire l'attention de toute la planète, il fallait une opération intégrée pour à la fois prévenir et combattre ce fléau. Effectivement, le sujet a été mis en avant ce qui, conséquemment, a rendu possible un débat, sept ans avant le coup d'envoi du

\footnotetext{
22 IBGE. Estimativas da população residente no Brasil e unidades da federação.

${ }^{23}$ FAUSTO. História do Brasil.

24 LOURENÇO; FONTES. Articulação e mobilização de parceiros.

${ }^{25}$ PORTAL DA COPA. Brasil prepara planos contra turismo sexual na Copa de 2014.
} 
FuLiA / UFMG - [...] deux facettes de la prostitution

Mondial. Néanmoins, il était clair que la CM 2014 devait être prise comme le point de départ d'un processus long puisque, traitant d'un tourisme caché et tentaculaire, une action forte mais unique ne serait pas assez efficace.

Pendant l'événement, une recherche approfondie à Recife a eu lieu pour mettre sur pieds les outils d'observation participante et des entretiens avec les porteurs du projet. En plus, des observations, ainsi que des conversations informelles, ont été réalisées notamment dans les quartiers où cette pratique se fait plus fréquemment. Bien que cette affaire soit délicate, particulièrement à l'égard des familles et habitants concernés, des témoignages importants ont été accordés même si, dans certaines situations, l'enregistrement des entretiens n'a pas été possible. Quant aux porteurs du projet, les entretiens se sont bien passés dans les deux commissariats - l'un au centre-ville et l'autre à l'aéroport - créés par la municipalité en association avec la police fédérale.

Un an après le Mondial, un deuxième contact a été établi avec les acteurs qui ont participé au premier recueil des données pour en parler d'avantage et notamment pour faire un bilan par rapport aux résultats attendus de la mise en place de cette politique. Certes, les chiffres ont montré une chute considérable concernant les plaintes déposées au commissariat, environ $45 \%$ de moins que la moyenne dans un événement majeur, mais, dans l'avenir, il y avait déjà quelques suspicions quant à la continuité du projet vu qu'un des commissariats avait déjà été fermé par manque de fonds publics.

En plus d'une politique publique efficace, les personnes impliquées ont conscience que, tant que le niveau éducatif de la région sera faible (résultat des inégalités sociales alarmantes), cette pratique aura toujours lieu plus ou moins fréquemment. Toutefois, il est légitime, non seulement de mettre en place des politiques pour combattre ce type de tourisme, mais, également, de profiter de l'ampleur de la CM 2014 pour discuter ce thème si cher à l'égard du football car, d'après certaines études, ${ }^{26}$ les événements de football attirent encore plus de visiteurs pratiquant le tourisme sexuel.

\footnotetext{
${ }^{26}$ BIRD; DONALDSON. "Sex, Sun, Soccer": Stakeholder-Opinions on the Sex Industry in Cape Town in Anticipation of the 2010 FIFA Soccer World Cup. BRACKENRIDGE; et al. Child Exploitation and the FIFA World Cup: A review of risks and protective interventions.
} 
FuLiA / UFMG - [...] deux facettes de la prostitution

\section{DISCUSSION}

Les études sur la relation entre la prostitution et les GES ont reçu une attention réduite jusqu'à maintenant et, en plus, normalement, ont une tendance d'analyse portée prioritairement sur les aspects économiques, laissant l'aspect social de côté. D’après Rao, « dans le monde universitaire, on n'a pas encore donné à la prostitution, y compris le tourisme sexuel, l'attention qu'ils méritent $»{ }^{27}$ De cette façon, cette investigation se penche sur la compréhension de ces questions chères à l'académie et propose une approche plus sociale dans le cadre des événements sportifs.

Hall et Selwood ${ }^{28}$ ont identifié une augmentation substantielle du nombre de prostituées en provenance d'Asie et d'Australie avant et pendant la Coupe de l’Amérique 1986 à Fremantle, ce qui a été attribué à une hausse de la demande par les visiteurs pour l'industrie du sexe. Bien que le gouvernement local ait su que ce problème existait, les lois et les règlements locaux ont été ajustés pour la durée de l'événement, ce qui a favorisé des pratiques illégales afin de répondre aux demandes des touristes en premier plan. Ainsi, plusieurs modifications sont accordées durant l'événement telles que l'ouverture des nouvelles boîtes de nuit, le changement de fonctionnement de celles-ci, entre autres. ${ }^{29}$

Selon Hall et Selwood,30 après la Coupe de l'Amérique 1986, « le gouvernement australien a mis en place plusieurs mesures afin de diminuer l'incidence des activités immorales résultant de l'organisation de l'événement ». Avant les J0 de 2004, une gamme de supports médiatiques (la BBC, l'AFP) a rapporté des protestations de certains pays d'Europe du Nord et de l'Église orthodoxe grecque contre les propositions faites par le maire d'Athènes afin d'augmenter le nombre de permis des maisons closes. Il faut souligner également que cela avait été le cas lors des J0 de

\footnotetext{
${ }^{27}$ RAO. Sex tourism in South Asia, p. 98.

${ }^{28}$ HALL; SELWOOD. America's Cup lost, paradise retained?, p. 110.

29 BIRD; DONALDSON. "Sex, Sun, Soccer": Stakeholder-Opinions on the Sex Industry in Cape Town in Anticipation of the 2010 FIFA Soccer World Cup.

${ }^{30}$ HALL; SELWOOD. America's Cup lost, paradise retained?, p. 112.
} 
FuLiA / UFMG - [...] deux facettes de la prostitution

Sydney en 2000.31 Plus récemment, en 2006, l'Allemagne avait également de nombreux centres de divertissement pour adultes au cours de la CM 2006, qui était très populaire auprès des visiteurs. ${ }^{32}$

Il est donc clair que le tourisme sexuel est une industrie, d'ailleurs, non seulement présente dans certains pays mais, dans le monde entier. En outre, dans certains pays comme Cuba, la Thaïlande et les Philippines, cette pratique peut être considérée comme centrale dans les affaires du tourisme local. ${ }^{33}$ En considérant les aspects liés aux GES, on observe que les pays hôtes doivent fréquemment faire face à un ajustement de leurs lois vis-à-vis des problèmes de la prostitution. ${ }^{34}$ Néanmoins, cela ne signifie pas que de telles mesures sont toujours effectuées afin d'enrayer cette pratique. En effet, dans la littérature académique, on trouve des cas divers quant aux enjeux politiques toujours en association avec l'aspect économique.

À cette question, par exemple, pendant l'organisation de la CM 2010 en Afrique du Sud, un débat a émergé au sujet de la légalisation de la prostitution et son potentiel économique à l'égard de l'événement. L'idée a d'abord été introduite par le chef de la police locale, Jackie Selebi, qui a insisté pour qu'une concession temporaire spéciale soit mise en œuvre pour libérer la prostitution et la consommation d'alcool en public, selon certains paramètres, pendant la durée de la CM 2010.35 Cette suggestion a été faite pour permettre une plus grande réglementation de l'industrie du sexe ce qui favoriserait la concentration de la police sur des crimes plus graves. ${ }^{36}$

Cette question apparaît dans les débats fondés sur les motifs économiques : à ce propos, la prostitution pendant la CM pourrait être rentable financièrement. Dans le cadre de la CM 2014 à BH, la mise en place du projet de " cours de langue " était conçue pour que les prostituées puissent, comme d'autres individus, profiter de

${ }^{31}$ GEHARMAN. The unfairness behind the Olympic Games fairplay.

32 BBC. Un an après la Coupe du monde 2014, seulement la moitié de l'héritage promis a effectivement été livré à la population.

${ }^{33}$ CLANCY. The Globalization of Sex Tourism and Cuba: A Commodity Chains Approach, p. 65.

${ }^{34}$ BESINGER. World Cup 2006: Sex trafficking and prostitution in Germany. CRAGGS; HENNING; LARSSON; LACZKO. Trafficking in human beings and the 2006 World Cup in Germany.

35 BIRD; DONALDSON. "Sex, Sun, Soccer": Stakeholder-Opinions on the Sex Industry in Cape Town in Anticipation of the 2010 FIFA Soccer World Cup.

${ }^{36}$ BLANDY. SA mulls prostitution law ahead of 2010. 
FuLiA / UFMG - [...] deux facettes de la prostitution

l'arrivée du Mondial. D’après Mme Bethânia, présidente de l'association, « si tout le monde profite de la CM, pourquoi ne pourrions nous pas en profiter aussi ? », et quant aux attentes, elle ajoute, « nous nous attendons à plus d'animation dans les boîtes de nuit, les hôtels, les saunas et dans les rues ». Sur la photo ci-dessous, des drapeaux brésiliens faisaient déjà le décor de l'entrée des maison closes du quartier avant le Mondial.

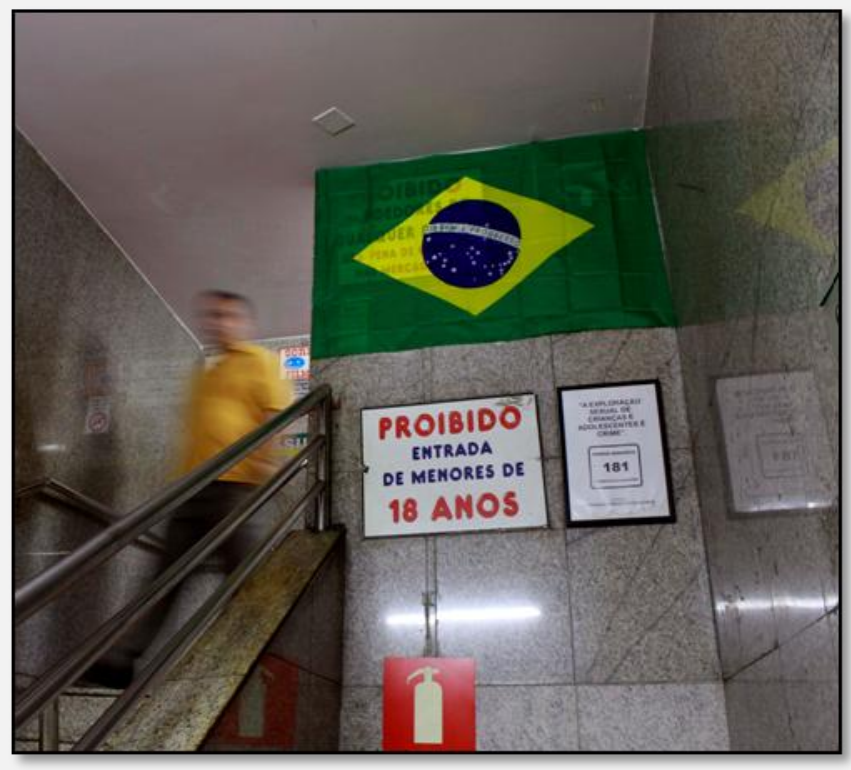

Photo 1 : Sur l'affiche :

" entrée interdite aux personnes de moins de 18 ans ».

En association avec ce côté économique du projet, la présidente souligne particulièrement son « but primordial de promouvoir la réglementation de la profession dans le pays $»{ }^{37}$ Même si la prostitution n'est pas un crime sur le territoire brésilien, il faut encore des améliorations d'ampleur pour que les conditions de travail des travailleuses du sexe soient jugées dignes. D’après Carla, prostituées depuis 10 ans, « la CM nous permet de parler de sujets tabous à l'égard de la société mais qui,

\footnotetext{
${ }^{37}$ Actuellement, un projet de loi pour la « réglementation de la profession » ( $n$ ํ 4211/2012) est en train d'être voté par la Chambre des députés brésilienne. D'après ce projet, toutes personnes ayant plus de 18 ans peuvent fournir des services sexuels en échange de rémunération. En plus, les personnes prostituées pourraient travailler comme autonome, collectivement dans les coopératives ou dans les maisons closes - qui seraient autorisées. Le projet de loi accorde également le droit de retraite spéciale après 25 ans. Bien évidemment, l'exploitation sexuelle et le proxénétisme demeurent interdits au Brésil.
} 
FuLiA / UFMG - [...] deux facettes de la prostitution

bon gré mal gré, doivent être mis en débat ». En outre, ces initiatives attirent l'attention des médias ce qui provoque une pression vis-à-vis des élus.

À ce sujet, la députée fédérale Jô Moraes, impliquée dans l'organisation de la CM, s'est exprimée lors de l'entretien : « même si, personnellement, je ne suis par pour la légalisation complète de la prostitution, il faut débattre ce thème urgemment. En plus de l'aspect du travail, il y a aussi diverses questions concernant la santé publique, car, une fois décriminalisée la profession, il est de la responsabilité du gouvernement de donner les conditions minimales d'hygiène et d'informations sur la protection contre les maladies infectieuses ». Aujourd'hui, au-delà de la prostitution, il y a également une préoccupation quant aux maladies sexuellement transmises ce qui n'empêche pas qu'une augmentation de la pratique se produit lors d'un GES. À ce sujet, d'après Clancy, ${ }^{38}$ même si en Afrique du Sud la prévalence du SIDA est l'un des plus élevés du monde avec environ 5,2 millions de personnes infectées, le tourisme sexuel y « prospère » toutes les années, notamment pendant les festivités.

À $\mathrm{BH}$, cette thématique fait partie d'une série de priorités de l'Association présidée par Mme Bethânia. «En plus de la distribution des préservatifs, on met l'accent sur la dangerosité des maladies infectieuses », dit-elle. Néanmoins, en ce qui concerne l'aide des pouvoirs publics, d'après les prostituées interrogées, il y a encore énormément de progrès à faire puisque, d'une manière générale, les élus sont absents à l'égard de la prostitution. En effet, la prostitution, même si on la considère comme le plus vieux métier du monde, reste peu comprise et peu soutenue par les institutions publiques.

Concernant le projet lui-même, selon la porteuse, au moment où les inscriptions ont été ouvertes, plus de 300 adhérentes se sont inscrites. Dans un premier temps, il était prévu d'enseigner trois langues étrangères - l'anglais, le français et l'espagnol - néanmoins, faute d'argent, l'Association a décidé de prioriser l'anglais puisque il s'agit, aujourd'hui, d'une langue presque universelle. Au fil du temps, l'adhésion des élèves a vertigineusement chuté et, d'après le professeur luimême, « même si les classes avaient une fréquence hebdomadaire, mes élèves ont

\footnotetext{
${ }^{38}$ CLANCY. The Globalization of Sex Tourism and Cuba: A Commodity Chains Approach, p. 75.
} 
toujours eu des problèmes par rapport à leur présence en classe ». En effet, lors d'une observation du terrain réalisée deux semaines avant la CM, cette problématique a été constatée dans la mesure où il n'y avait que douze participantes.

Toutefois, même si le nombre d'élèves n'était pas suffisant d'un point de vue extérieur, celles qui ont suivi cette formation ont apprécié. En plus, d'après l'Association, il y a, à présent, une volonté que le projet continue même après l'événement. Bien que l'aspect économique soit toujours un argument fort à l'égard des projets conçus, il est évident, à partir des données collectées, que l'instruction des adhérentes est une priorité à long terme. Effectivement, l'avenir des adhérentes joue en rôle décisif au sein des débats dans l'association et, par conséquent, un meilleur niveau d'instruction générale est envisagé car cela permettrait des nouvelles possibilités d'emplois.

Les «professionnels du sexe » demeurent toujours en marge de la société brésilienne. Cependant, de telles initiatives sont essentielles pour restaurer ce débat afin que des améliorations soient apportées dans leur vie. En outre, la prostitution amène d'autres problèmes sociaux extrêmes et cela doit être mis en exergue notamment dans le cadre des événements sportifs majeurs. Parmi ces problématiques, il faut mettre en évidence la question du tourisme sexuel infantile et la traite des êtres humains.

L'Organisation mondiale du tourisme (OMT) définit le tourisme sexuel comme " voyages effectués à l'intérieur du secteur du tourisme, ou de l'extérieur de ce secteur, mais en utilisant ses structures et ses réseaux, avec le but principal de promouvoir une relation sexuelle commerciale entre les touristes et les résidents locaux ». ${ }^{39}$ Par conséquent, « le tourisme sexuel est une rencontre purement physique dans laquelle le partenaire n'est plus qu'un objet animé ».40 La perception traditionnelle est que les touristes (généralement des hommes) voyagent dans les pays en développement dans le but d'avoir des relations sexuelles avec des prostituées. ${ }^{41}$ Néanmoins, dans le tourisme sexuel, on trouve, évidemment, le

\footnotetext{
${ }^{39}$ KATERERE. Female sex tourism lurks beneath Cape Town's surface, p. 12

${ }^{40}$ RAO. Sex tourism in South Asia, p. 98.

${ }^{41}$ DE MOYA; GARCIA; HEROLD. Female Tourists and Beach Boys: Romance or Sex Tourism?.
} 
FuLiA / UFMG - [...] deux facettes de la prostitution

phénomène de l'exploitation sexuelle des enfants et des adolescents. Au Brésil, cette pratique existe et ne résulte pas d'un seul facteur. Il s'agit d'un problème complexe et, selon chaque région, des particularités doivent être mises en évidence pour la comprendre. ${ }^{42}$ D'après les études, la pauvreté est l'un des principaux facteurs de vulnérabilité et, en dépit d'être la septième économie du monde, le Brésil a encore 16,27 millions de personnes vivant dans l'extrême pauvreté - environ 8,5\% de la population totale. ${ }^{43}$

Parmi les cinq régions du Brésil, le Nord-est est celle qui présente le plus grand nombre de dossiers relatifs à l'exploitation des enfants avec plus de $30 \%$ du total. Selon une étude publiée, ${ }^{44}$ la violence sexuelle infantile peut être classée en trois catégories : l'abus sexuel (avec 70\% des cas), l'exploitation sexuelle (avec 28\%) et la pornographie infantile (avec $2 \%$ ). De cette façon, la mise en place d'une politique publique à Recife - l'une des principales villes du Nord-est - s'avèrerait primordiale pour combattre réellement ce crime. D’après l'un des policiers fédéraux interrogé, « le fait que la ville se situe au bord de la mer favorise l'exploitation sexuelle. En outre, garçons et filles cherchent cette activité comme un moyen de survie et, généralement, cette relation avec le touriste étranger les font rêver d'une vie meilleure dans un autre pays. Néanmoins, cela n'arrive presque jamais ! Dans les villes côtières, cette exploitation sexuelle est directement liée au tourisme. »

En tenant compte de la complexité du problème, les porteurs du projet ont effectué une carte du tourisme sexuel dans la région en association avec d'autres institutions impliquées dans ce thème. Ainsi, à partir de 2009, soit 5 ans avant le Mondial, les zones d'actions étaient déjà choisies pour entamer la première partie du projet qui prévoyait un rapprochement avec les habitants concernés afin de prévenir l'entrée des nouveaux enfants dans ces réseaux. D’après Bird et Donaldson, il y a eu des inquiétudes similaires concernant la CM 2010 car des enfants et des organisations des droits de l'homme ont « averti que l'exploitation sexuelle infantile pouvait subir

\footnotetext{
${ }^{42}$ LOURENÇO; FONTES. Articulação e mobilização de parceiros.

43 IBGE. Estimativas da população residente no Brasil e unidades da federação.

${ }^{44}$ LOURENÇO; FONTES. Articulação e mobilização de parceiros.
} 
un accroissement périlleux dans le pays hôte avant le coup d'envoi du Mondial, avec des femmes et des enfants victimes de violence et qui seraient forcés d'entrer dans l'industrie du tourisme sexuel ». 45

Cette mesure vis-à-vis des habitants locaux s'avère d'autant plus efficace que, dans le contexte brésilien, cette exploitation n'est pas perçue comme un crime. En effet, au cours de l'enquête de terrain, à travers des conversations informelles, on a pu constater qu'une partie de la population regarde cette activité comme une opportunité de changement de vie. Dans certains cas, on observe le consentement des parents ce qui rend cette bataille encore plus difficile. En plus, les coordinateurs des réseaux, ceux qui en profitent le plus, se servent de cette « promesse de changement de vie » pour attirer encore plus d'enfants dans cette activité.

Au centre-ville, l'endroit le plus touristique et où se situait la FAN FEST organisée par la FIFA, au-delà des policiers présents, des affiches sur le sujet de l'exploitation sexuelle infantile ont été déployées mettant l’accent sur la prévention de ce crime.

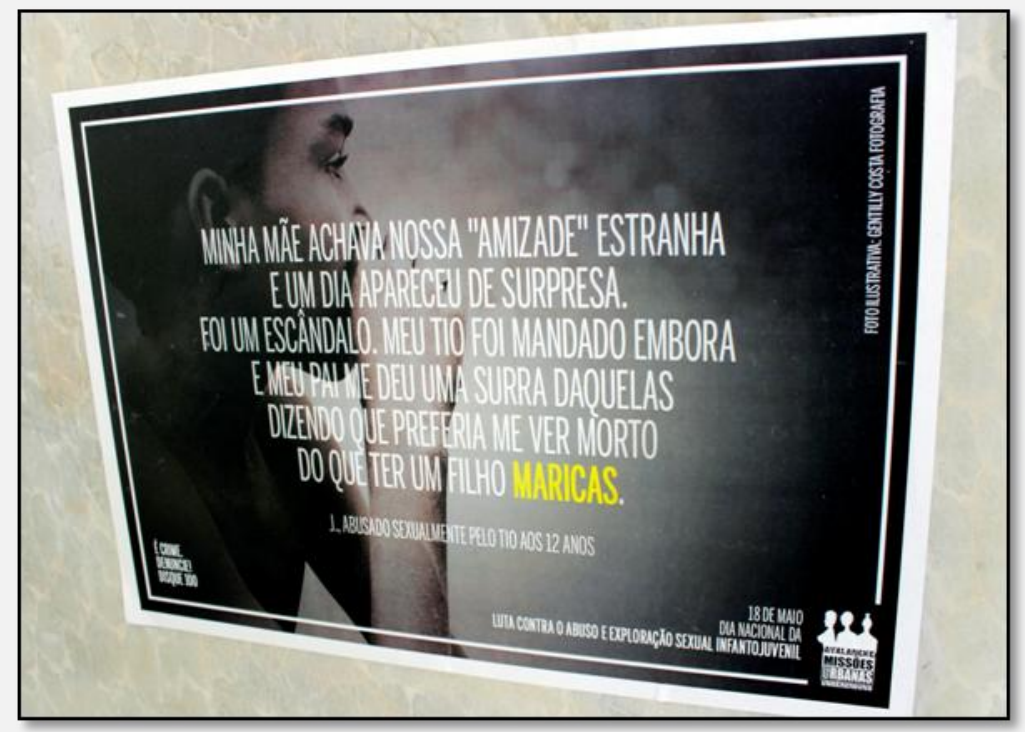

Photo 2 : Sur l'affiche : « ma mère croyait que notre amitié était très étrange et un jour elle a fait irruption. Ça a été un scandale ! Mon oncle a été jeté dehors et mon père m'a donné une raclée en disant qu'il préférait me voir mort que d'avoir un fils pédé. »

* Confession d'un enfant abusé de 12 ans.

${ }^{45}$ BIRD; DONALDSON. "Sex, Sun, Soccer": Stakeholder-Opinions on the Sex Industry in Cape Town in Anticipation of the 2010 FIFA Soccer World Cup, p. 35. 
Cette affiche montre comment cette exploitation est enracinée dans la culture des habitants. Même dans leur vie quotidienne, les enfants sont toujours victimes des adultes et exposés aux actes de violence. Ces détails concernant les différents acteurs de cette pratique rend l'action des pouvoirs publics encore plus complexe mais, au même moment, réaffirme la nécessité d'une politique efficace.

Alors, les porteurs du projet ont décidé d'intervenir dans trois domaines différents : dans les quartiers défavorisés de Recife - auprès des habitants locaux -; dans les réseaux touristiques impliqués; et, une troisième action, qui prévoyait la mise en place de deux commissariats spéciaux - à l'aéroport et au centre-ville ouverts $24 / 24 \mathrm{~h}$, pour lutter contre cette pratique le plus efficacement possible. En réalité, durant le Mondial, les commissariats ont été très vivants puisqu'ils étaient implantés dans les endroits les plus ciblés par les touristes.

Quant au rapprochement des habitants locaux, il est clair que cette politique ne sera pas vraiment suffisante face à la complexité du défi. Seul un changement de mentalité, provoqué par une politique éducative à long terme, pourrait améliorer la compréhension de l'exploitation sexuelle. Alors que le seuil de pauvreté demeure si précaire, l'offre d'argent dans le monde du tourisme sexuel aura toujours raison des résistances. À ce sujet, les interviewés n'ont pas manqué de critiquer l'absence d'intervention des pouvoirs publics. À présent, faute de fonds publics, la municipalité a déjà fermé le commissariat même si cette politique avait réussi à diminuer le nombre de dossiers à l'égard du tourisme sexuel de $45 \%$, selon les policiers.

Comme s'il n'y avait pas assez de problèmes liés au tourisme sexuel infantile, cette pratique pourrait conduire à d'autres ennuis, tels que la traite des êtres humains. Avant la CM 2006, il y avait un rumeur dans les médias internationaux selon laquelle environ 40000 femmes et enfants pourraient être victimes de cette traite, afin de répondre à la demande touristique en Allemagne. ${ }^{46} \mathrm{Au}$ Brésil, malgré le manque d'informations précises sur ce thème, la police fédérale, dans le cadre de la CM 2014,

\footnotetext{
${ }^{46}$ BENSINGER. World Cup 2006: Sex trafficking and prostitution in Germany. TAVELLA. Sex trafficking and the 2006 World Cup in Germany: concerns, actions and implications for future international sporting events.
} 
FuLiA / UFMG - [...] deux facettes de la prostitution

était également attentif quant à la traite des êtres humains. Néanmoins, l'accent a été mis sur le tourisme sexuel infantile.

\section{ConCluSion}

L'arrivée de grands événements sportifs génère une grande attente dans la population locale du pays hôte à l'égard de possibles améliorations des conditions de vie dues aux changements promis par les élus. Typiquement, ces événements sont considérés comme accélérateur de transformations sociales déjà nécessaires et peuvent, grâce à la visibilité insufflée par cette occasion, attirer des investissements robustes afin que ces changements se concrétisent. Au Brésil, ces attentes étaient d’autant plus élevées que le pays est considéré par le classement des Nations Unies comme l'uns des pays les plus inégaux du monde au niveau social.

D'après les premiers bilans publiés dans la presse nationale et internationale, l'organisation du Mondial a été un succès incontestable et la peur qui dominait les organisateurs quant au bon fonctionnement des équipements sportifs et des transports en commun a été supplantée par la capacité de la population brésilienne à accueillir ces visiteurs. Cette excitation a atteint un tel point que le président de la FIFA, Joseph Blatter, a déclaré à plusieurs reprises que « la CM 2014 a été la plus grande fête du football jamais vue ${ }^{47}$ Néanmoins, en ce qui concerne les héritages économiques, le bilan s'avère catastrophique, selon les premiers documents. En effet, le coût du tournoi a énormément dépassé le budget initial, plusieurs projets d'infrastructures n'ont pas été accomplis, certains stades construits ont un avenir incertain, entre autres.

Au sujet des héritages sociaux, les changements attendus ont également été en deçà des attentes. En outre, d'une manière générale, pendant les sept années d'organisation de l'événement, les responsables étaient tellement absorbés par les délais relatifs aux travaux d'infrastructures que les projets sociaux n'ont pas été pris

${ }^{47}$ ITRI. Um ano após a Copa 2014, oito estádios apresentam prejuízos financeiros. 
au sérieux ce qui a affaibli encore plus les legs prévus dans ce domaine. Toutefois, même dans une situation si catastrophique, les deux projets présentés dans cette étude sont parvenus à une certaine visibilité et, de façon plus décisive, ont relancé le débat sur la prostitution et le tourisme sexuel infantile. Tant au niveau de la société qu'au niveau académique, ces problématiques méritent plus de place pour que la situation des personnes impliquées puisse s'améliorer vis-à-vis des préjugés et des autres nuisances qui y sont associés.

Bien que les bilans sociaux de la CM 2014 soient faibles, l'action de l'Association des prostituées de Belo Horizonte et de la municipalité de Recife, en association avec la police fédérale, sont des exemples réussis de politiques publiques efficaces. Certes, il faut admettre qu'à long terme d'autres innombrables actions doivent être mises en place mais, à court terme, les résultats sont favorables.

En conclusion, cette étude ambitionne également de signaler une tendance dans le milieu universitaire à discuter le thème de la prostitution par le biais économique et en la reléguant fréquemment à un aspect négatif par rapport aux GES. Nos résultats permettent un autre regard sur cette question, en particulier en ce qui concerne le tourisme sexuel infantile. Malgré les absurdités qui entourent ce fléau, il est toujours possible de découvrir des politiques publiques originales qui visent l'éradication de ce genre d'exploitation.

\section{BibLIOGRAPHIE}

ALMEIDA, Marco Antonio Bettine de; GUTIERREZ, Gustavo Luis. O lazer no Brasil: do nacional-desenvolvimentismo à globalização. Conexões, Campinas, v. 3, n. 1, p. 36-57, 2005.

AMORA, Dimmi. Um ano após a Copa do Mundo, 35 obras não estão prontas. Folha de São Paulo, São Paulo, 7 jun. 2015. Disponível em: https://goo.gl/9qB9C5. Acesso em: 06 nov. 2015.

BARGET, Eric; GOUGUET, Jean-Jacques. L'accueil des grands événements sportifs: quel impact économique ou quelle utilité sociale pour les régions ? : 
l'exemple de la Coupe du monde de Rugby 2007 en France. Région et Développement, [s. I.], v. 31, p. 93-117, 2010.

BBC. Un an après la Coupe du monde 2014, seulement la moitié de l'héritage promis a effectivement été livré à la population. BBC, [s. I.], 2015.

BENSINGER, Gad. World Cup 2006: Sex trafficking and prostitution in Germany. Crime \& Justice International, Estados Unidos da América, v. 22, n. 95, p. 19-21, nov.-dez. 2006.

BIRD, Ruth; DONALDSON, Ronnie. "Sex, Sun, Soccer": Stakeholder-Opinions on the Sex Industry in Cape Town in Anticipation of the 2010 FIFA Soccer World Cup. In: URBAN FORUM. Chicago: 2009. p. 33-46.

BLANDY, Fran. SA mulls prostitution law ahead of 2010. Mail and Guardin Online, África do Sul, 22 jun. 2009. Disponível em: https://goo.gl/YpzKsj. Acesso em: 18 Jan 2015.

BRACKENRIDGE, Celia et al. Child Exploitation and the FIFA World Cup: A review of risks and protective interventions. Londres: Brunel University London, 2013.

CHARRIER, Dominique; JOURDAN, Jean. L'impact touristique local des grands événements sportifs: une approche qualitative de la Coupe du monde de Rugby en Île-de-France. Téoros, v. 28, n. 2, p. 45-54, 2009.

CLANCY, Michael. The Globalization of Sex Tourism and Cuba: A Commodity Chains Approach. Studies in Comparative International Development, [s. I.], v. 36, n. 4, p. 63-88, 2002.

CRAGGS, Sarah; HENNING, Jana; LARSSON, Fred; LACZKO, Frank. Trafficking in human beings and the 2006 World Cup in Germany. [s. I.]: International Organization for Migration, 2006. 28 p. Rascunho de relatório.

DAMO, Arlei Sander; OLIVEN, Ruben George. O Brasil no horizonte dos megaeventos esportivos de 2014 e 2016: sua cara, seus sócios e seus negócios. Horizontes Antropológicos, Porto Alegre, ano 19, n. 40, p. 19-63, jul.-dez. 2013.

DE MOYA, Tony; GARCIA, Rafael; HEROLD, Edward. Female Tourists and Beach Boys: Romance or Sex Tourism? Annals of Tourism Research, Grã-Bretanha, v. 28, n. 4, p. 978-997, 2001.

FAUSTO, Boris. História do Brasil. 14. ed. São Paulo: Edusp, 2012.

FREDLINE, Elizabeth. Host and Guest Relations and Sport Tourism. Sport, Culture and Society, [s. I.], v. 8, n. 2, p. 263-279, 2005.

GEHRMAN, Craigg. The unfairness behind the Olympic Games fairplay. Newropeans MAGAZINE, [s. I.]. 2 fev. 2017. Disponível em: https://goo.gl/vvX9zQ. Acesso em: 1 dez. 2017.

GETZ, Donald. Festivals, Special Events, and Tourism. New York: Van Nostrand Reinhold, 1990. 
HALL, Michael; SELWOOD, H. J. America's Cup lost, paradise retained? In: SYME, Geoffrey J; SHAW, B. J.; FENTON, D. Mark; MUELLER, Walter S. The planning and evaluation of hallmark events. Swansea: Gower Pub Co. Itd, 1989.

HALL, Michael. Adventure, sport and health tourism. In: WEILER, Betty; HALL, Michael. Special interest tourism. London: Belhaven Press, 1992. p. 141-158.

HIGHAM, James. Sport as an Avenue of Tourism Development: An Analysis of the Positive and Negative Impacts of Sport Tourism. Current Issues in Tourism, [s. I.], v. 2,. n. 1, p. 82-90, 1999.

IBGE. Estimativas da população residente no Brasil e unidades da federação. Brasília: Instituto Brasileiro de Geografia e Estatística, 2014.

ITRI, Bernardo. Um ano após a Copa 2014, oito estádios apresentam prejuízos financeiros. Folha de São Paulo, São Paulo, 16 jun. 2015. Disponível em: https://goo.gl/Nia469. Acesso em: 18 jan. 2016.

JUNOD, Thomas. Grands événements sportifs: des impacts multiples. Finance et Bien Commun, [s. I.] v. 26, p. 92-98, 2007.

KATERERE, Frederic. Female sex tourism lurks beneath Cape Town's surface. The Big Issue, London, p. 10-15, 2007.

KLEIN, Marco Aurélio. Futebol brasileiro: 1894 à 2001. São Paulo: Escala, 2001.

LOURENÇO, Marisa; FONTES, Miguel. Articulação e mobilização de parceiros. Brasília: SESI-CN; Geração Editorial, 2014.

MADER, Helena. Lei da prostituição divide a Câmara. Correio Braziliense, p. 6, 27 jan. 2013.

MADRIGAL, Robert.; BEE, Colleen; LABARGE, Monica. Using the Olympics and FIFA World Cup to enhance global brad equity. In: AMIS, John M.; CORNWELL, T. Bettina. Global Sport Sponsorship, Oxford: Berg, 2005, p. 179-190. (Sport Commerce and Culture).

MATHIESON, Alister; WALL, Geoffrey. Tourism: Economic, Physical and Social Impacts. Londres: Longman, 1982.

OLSEN, Marvin; MERVIN, Donna. Towards a methodology of conducting social impacts assesments using quality of life indicators. In: FINSTERBUSH, Kurt; LLEWELLYN, Lynn G; WOLF, C. P. Methology of social impact assessment. Pênsilvania: Dowden, Hutchinson \& Ross, 1977, p. 43-63.

PORTAL DA COPA. Brasil prepara planos contra turismo sexual na Copa de 2014. Agência Brasil, São Paulo, 03 out. 2010. Disponível em: www.portaldacopa.org.br. Acesso em: 15 ago. 2015.

RAO, Nina. Sex tourism in South Asia. International Journal of Contemporary Hospitality Management, v. 11, n. 2/3, p. 96-99, 1999.

RITCHIE, J. R. Brent. Assessing the impact of hallmark events: conceptual and reserach issues. Journal of travel research, v. 23, n.1, p. 2-11, jul. 1984. 
ROBERTS, Ken. The leisure industries. Basingstoke: Palgavre Macmillan, 2004.

SOUSA, Fernando J. Pires de. Comprendre les récentes manifestations de rue. Problèmes économiques, n. 3085, p. 5-12, 2014.

TAVELLA, Anne Marie. Sex trafficking and the 2006 World Cup in Germany: concerns, actions and implications for future international sporting events. Northwestern University Journal of International Human Rights, v. 6, p. 196-217, 2007.

WAITT, Gordon. Social impacts of the Sidney Olympics. Annals of Tourisma Research, v. 30, n. 1, p. 194-215, 2003.

Recebido para publicação em: 18 jan. 2017. Aprovado em: 26 maio 2017. 